\title{
Research on user Perception behavior of Mobile Library Based on Technology Acceptance Model
}

\author{
Sun Qizhi \\ Library of Zaozhuang University, Zaozhuang, Shandong, 277160, China \\ email: zzsunqizhi@163.com
}

Keywords: Mobile Library, User Perception Behaviour, Technology Acceptance Model, Mobile Service Quality

\begin{abstract}
In recent years, Chinese scholars have paid attention to the cognitive behavior of portable library users, and adopted the technology acceptance model (TAM) as the theoretical basis for research. In addition to traditional Tam variables (such as perceived usefulness, ease of use, and intention to act), other external and internal variables describe and validate user perceptions to change the degree. In order to understand the research progress of users' cognitive behavior of China Mobile Library, this paper focuses on the construction and expansion of TAM model, potential variables, summary and discussion of empirical research. This paper puts forward the research of user behavior in order to predict and describe user behavior dynamically and improve the quality of Library mobile service.
\end{abstract}

\section{Introduction}

Mobile library is the expansion of digital library service in the ubiquitous environment. On the one hand, its successful establishment and application depend on advanced technology, on the other hand, users' behavior feedback on mobile library [1]. In the aspect of the development of mobile information service, the utilization rate of mobile library is low and it is difficult to accept the use of users. The methods of changing users' cognitive attitude towards mobile library, and the methods of determining and influencing the dominant factors of users' acceptance in order to further improve the quality of mobile service of library are all related to the research of users' cognitive action. In the case of promoting the mobile service of the library, the users of the mobile library should know and accept, grasp the guidance based on the user's feedback timely and accurately, and construct the important codes of the success or failure of the mobile library. In order to master the technology of mobile library users and the factors that influence the technology popularization of mobile library users, it is necessary to show the library services. When CNKI files are used as data sources, the user perception of mobile library is used to make relevant statistics for action research, and the key words "mobile library", "user cognitive action", "technology acceptance model" and "collection" are used [2]. A total of 81 documents were searched. (as of January 2017), the cognition and acceptance of mobile library have different perspectives from multiple levels of research and development users, lion king. In order to build, expand, potential variables, potential variables, empirical research, improve the detailed analysis of follow-up research, focus on more dynamic user behavior prediction, explain, improve the mobile service quality of library.

\section{TAM Model Overview}

In 1989, Davis put forward the TAM based on the theory of rational behavior. When users use their technology to improve performance and recognition, technology is considered to be useful and convenient to use. Use technology trend, tend to use the strong will, and then, finally, this is technology acceptance trend. Tam publishes a set of common factors that affect users' acceptance of information system. These factors can effectively explain and predict users' attitudes and behaviors towards information system users. With the development of research, the more experienced 
application of technology acceptance model is a powerful tool to explain and predict the user's information technology acceptance behavior [3]. The theory of technology acceptance model developed from TAM, Tamm 2, UTAUT to TAML 3. Technology acceptance model is a more reasonable model, which provides a main line for the follow-up research. That is, the user's usage depends on the user's behavior intention. The technology acceptance model of mobile library users' cognition action is applicable to the analysis, the investigation of various problems faced by the library users in the acceptance and utilization of information services in the mobile service environment, the development of mobile information services and the development of the improvement role of the construction library and the improvement of the contribution library mobile service quality, which is far from enough.



Figure 1 Vector dynamic model of digital library research in the era of big data

\section{Construction and Extension of TAM Model in Mobile Library}

In recent years, scholars in the field of Library informatics try to expand and modify the model to further explain user behavior by combining various user behavior theories and models with the new information environment. Based on the study of acceptance model, most scholars introduce relevant variables, build a model and expand the perception behavior of mobile library users[4]. Fig. 1 shows a specific path. Scholars mainly explain the external variables from three perspectives to predict and predict the cognitive behavior of users. Results; Take external variables as useful old variables, such as perceived usefulness, perceived joy, relevance, screen design, domain knowledge, personal characteristics, self-efficacy. At the same time, gender, age, education and other adjustment variables are introduced to clarify the relationship between independent variables and dependent variables. It reveals the acceptance, adaptability and importance of different groups to mobile library. In addition, in the process of studying the audience of information technology, the objective observation of the actual action is the most ideal. However, considering the difficulty of measurement, the current domestic literature believes that there is a positive correlation between action intention and actual use of action, and action intention as the final dependent variable instead of use of action.

\subsection{Core Variable}

In order to study the influencing factors of users' attitude and behavior intention to mobile database, scholars applied Tam theory framework in the field of mobile database. The research focuses on perceived usefulness, ease of use, action attitude and action intention[5]. Variable expansion TAM model suggests that perceived usability and ease of use affect user attitudes. Perceived ease of use affects the user's intention of action by influencing perceived usefulness. Compared with traditional library, mobile library breaks the limitation of time and space. In order to get the information resources that users need, the library is widely unable to connect, the mobile users know the usefulness of the library, and the working performance of the mobile library is improved, which can improve users' action intention, while the mobile library system platform is easy to operate. Simple and practical, friendly screen interface. At the same time, enhance the user's 
understanding of the usability of mobile library, and affect its perceived usability. In this way, there will be a positive attitude towards mobile library services.

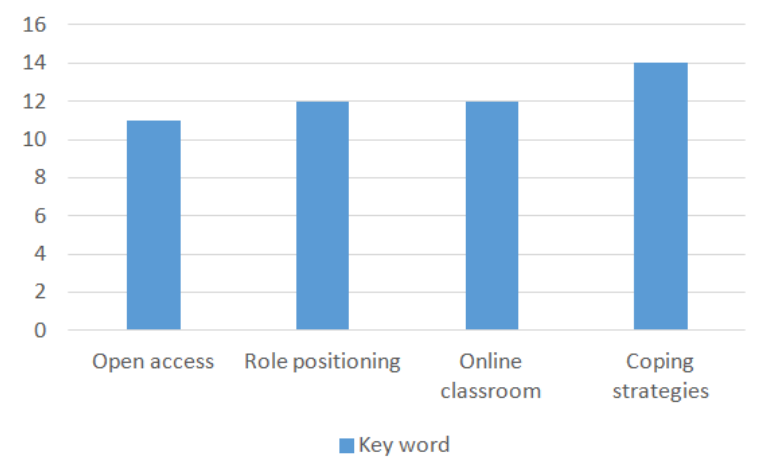

Figure 2 Keyword statistics

\subsection{Variables Affecting Perceived Usefulness}

The current variables that affect perceived usefulness are network externality, social impact, system characteristics and information quality [6]. The theory of network externality shows that network externality will affect perceived usefulness and ease of use. In order to establish the user acceptance model of Liu Yanfang's Portable Library, the network externality variable is introduced. Wang Shuang pointed out that the mobile library is new, and users did not touch it before using it for the first time. To this end, we should first observe the actions and tendencies of others, and be influenced by the mass media and the people around us. The usefulness and ease of use of perception can not be ignored.

Table 1 List of the number of students and total cost of teaching in a Provincial College of traditional Chinese medicine during the period of the 12th Five Year Plan

\begin{tabular}{|c|c|c|}
\hline Student nature & 2010 & 2015 \\
\hline This specialty & 12205 & 129811 \\
\hline Graduate student & 1824 & 2610 \\
\hline State Education Students & 741 & 564 \\
\hline Adult education students & 1907 & 1737 \\
\hline Student aggregate & 16675 & 17895 \\
\hline Total cost of teaching & RMB 573.44 million & RMB 766.58 million \\
\hline
\end{tabular}

\subsection{Variables Affecting Perceived Ease of Use}

Based on the comprehensive literature research, the variables that affect the convenience of use are mainly network externality, social impact, system characteristics, interface characteristics, individual innovation and personal differences (domain knowledge, self-efficacy). Lin Qiang uses the externality of the network as the internal variable of social influence factors, and establishes the user model of mobile database together with other variables. Social influence is the main factor that influences the user of Han super mobile service to adopt action integration model [7]. The interface characteristics of information system can be divided into terminology, screen design and navigation. Since then, Tam based library research has been following this standard, I believe that the interface function will affect the convenience of digital library.

\section{Empirical Research}

At present, the research purposes of mobile library users' cognitive behavior in China are divided into college students, graduate students (including doctoral students) and university teachers. As the research object, it is easy to conduct a questionnaire survey with university teachers and students as the object, which can not only collect sample data, but also save research costs [8]. At the same time, as the main users of mobile database, university teachers and students are very representative. 
As a highly educated group, university teachers and students have rich domain knowledge and strong self-efficacy. Their use of mobile library is also the main service target of mobile library. Most of the researches related to the perceptual behavior of library users have changed from qualitative and descriptive statistical research to quantitative and empirical research. Most scholars use rebound level 5 or 7 scale to design the questionnaire. In order to complete the survey, before the implementation of the questionnaire survey, we usually conduct a sampling survey, collect paper data through paper or web questionnaires, analyze the data, and study the influencing factors that affect the use of portable library users.

\section{Conclusion}

User's understanding of mobile library is a more complex operation [9]. The follow-up research on the perception behavior of mobile library must be objectively analyzed from multiple dimensions, and the dominant factors that affect the users' acceptance and use of mobile library must be systematically and comprehensively grasped. In the future, we must pay attention to the following issues in the research of user cognitive behavior based on TAM mobile database. The current empirical research on the perception behavior of mobile library users is the virtual relationship theory between dependent variables and independent variables. The composition of influencing factors and their various complex relationships may not be fully described. There are specific defects and deviations. In the future research, the appropriate addition or deletion of several variables can be different, in various cases, the subdivision variables of group and deceleration, the expansion of several potential variables and external variables in TAM model or the expansion of more variables between variables. This is a subtle relationship, comparing and analyzing whether the cognitive behavior of mobile library users is quite different in different fields and user groups. Most of the current researches related to users' perceptual behavior use profile research method to analyze the relationship between variables, that is, to take a sample at a specific time, and to make statistical analysis on the selected samples. The empirical conclusion can explain the respondents at a certain time [10]. The Static usage of a point cannot fully reflect the whole usage process. Neglecting the process will inevitably affect the generality of the research conclusion. The research on user perception behavior in mobile database should be a long-term dynamic process. At least 2 times of sampling are required, and there must be a certain interval between each sampling time. In the future, in order to dynamically track the user's behavior, the system log and two-way self-report will be compared to grasp the actual user behavior, introduce the construction of mobile library, improve the mobile service quality of the library, and add long-term observation and sampling survey for more fields.

\section{References}

[1] Tenriwaru, Amiruddin A, Pontoh G T. The Impact of Behavior of Tax Inspection Report Application User on Tax Performance Approach Technology Acceptance Model (TAM) on Tax Office Madya Makassar, 2017.

[2] Chen C F, Xu X, Arpan L. Between the technology acceptance model and sustainable energy technology acceptance model: Investigating smart meter acceptance in the United States, vol. 25, pp. 93-104, 2017.

[3] Asghar Ehteshami. Barcode Technology Acceptance and Utilization in Health Information Management Department at Academic Hospitals According to Technology Acceptance Model. Acta Informatica Medica, vol. 25, no. 1, pp. 4, 2017.

[4] Fiedler M, Arlos P, Gonsalves T, et al. In press: Time is perception is money - web response times in mobile networks with application to Quality of Experience, vol. 6821, pp. 179-190, 2017.

[5] Munique Peleias, Patricia Tempski, Helena BMS Paro,. Leisure time physical activity and quality of life in medical students: Results from a multicentre study. Bmj Open Sport — Exercise 
Medicine, vol. 3, no. 1, pp. e000213, 2017.

[6] Niti Mittal, Monica Chaudhary, Shirin Alavi. Development and Validation of Teachers Mobile Learning Acceptance Scale for Higher Education Teachers. International Journal of Cyber Behavior, Psychology and Learning (IJCBPL), vol. 7, 2017.

[7] Guang Chen, Shu Liu, Gereon Hinz,. Deep Anticipation: Light Weight Intelligent Mobile Sensing in IoT by Recurrent Architecture. IET Intelligent Transport Systems, 2017.

[8] Letizia Mortara, Nicolas Parisot. How do fab-spaces enable entrepreneurship? Case studies of 'makers' - entrepreneurs. International Journal of Manufacturing Technology \& Management, vol. 32, no. 1, pp. 16, 2018.

[9] Aidin Namin. Revisiting customers' perception of service quality in fast food restaurants. Journal of Retailing \& Consumer Services, vol. 34, pp. 70-81, 2017.

[10] Santos P R D, Meneghim M D C, Ambrosano G M B, et al. Influence of quality of life, selfperception, and self-esteem on orthodontic treatment need, vol. 151, no. 1, pp. 143-147, 2017. 